\title{
Extraretinal information about eye position during involuntary eye movement: Optokinetic afternystagmus
}

\author{
HAROLD E. BEDELL, JEANNE F. KLOPFENSTEIN, and NANYONG YUAN \\ University of Houston, Houston, Texas
}

\begin{abstract}
Despite importance for theories of perception, controversy exists as to whether information is available to the perceptual system about involuntary as well as voluntary eye movements. We measured the perceived direction of targets flashed briefly in an otherwise dark field during the primary phase of optokinetic afternystagmus (OKAN), an involuntary eye movement that persists in darkness following optokinetic stimulation. Perceived direction was measured by unseen pointing in one experiment and by pointing made under visual control in a second experiment. Pointing was essentially veridical in both experiments, indicating that accurate extraretinal information about eye position (presumably, as efference copy) exists for OKAN. Illusory motion of visual targets, which can occur during involuntary oculomotor responses, therefore cannot be attributed to a lack of efference-copy signals for such eye movements.
\end{abstract}

It has long been recognized that the perceived direction of a visual target depends upon the retinal locus stimulated by the target's image and concurrent information about the position of the eyes in the head (Gruesser, 1986; Wade, 1978). For example, a target's retinal-image position can change because either the target or the eyes move. For these two situations to be distinguished, as they readily are under most conditions, information in addition to the target's retinal-image location is required. Retinal information provided by a structured visual field has been shown to exert substantial influence on the perceived direction of visual targets (see Howard, 1982, for a review; see also Matin et al., 1982; Stark \& Bridgeman, 1983); obviously, this source of information is ineffective in reduced-cue situations. In such situations, and probably under normal viewing conditions as well, perception of a target's direction depends upon extraretinal information about eye position.

Potential sources of eye-position information are (1) internal neural copies of efferent commands (efference copy), and (2) afference from receptors in the extraocular muscles and tendons. Research indicates that, relative to efference copy, the role of muscle afference is relatively minor (Guthrie, Porter, \& Sparks, 1983; Skavenski, Haddad, \& Steinman, 1972).

Although the concept was anticipated by earlier authors (see Gruesser, 1986, for a review), von Holst and Mittel-

This study was supported by Research Grant R01-EY05068 and Research Training Grant T35-EY07088 from the National Eye Institute. Part of the results were presented at the 1988 ARVO meeting. We thank Chris Kuether for assistance in the design and construction of the OKN drum, John Feaster for statistical consultation, and Chris Johnson and Bob Post for helpful suggestions on the manuscript. Correspondence may be addressed to Harold E. Bedell, College of Optometry, University of Houston, Houston, TX 77204-6052. staedt (1950/1971) provided a theoretical framework for the interaction between retinal signals of target location and efference-copy information about eye position in the perception of visual direction and motion. Their model successfully accounts for the constancy of visual direction during normal voluntary eye movements and for motion of the visual world that is perceived when intended eye movements are prevented (e.g. , by extraocular muscle paralysis) or when eye movements are generated passively (e.g., by tapping on the eye with a finger). The model also correctly predicts that an afterimage is perceived to move in darkness during voluntary eye movements. It is noteworthy that von Holst and Mittelstaedt postulated that efference copy originates at a low level in the motor pathway, and stated explicitly that such efference-copy activity occurs "not only for the so-called 'voluntary' movements, but also for involuntary movements ... as when the head and body are turned (labyrinthine nystagmus) or the visual world moves before us (optomotor nystagmus)"' (p. 54). As evidence that involuntary eye movements are accompanied by an efference-copy signal, they reported observations that an afterimage, viewed in darkness, appears to move during vestibular nystagmus. This observation has also been reported by other authors (Goethlin, 1946; Graybiel \& Hupp, 1946; Morrison, 1984), but so has the contrary observation-of perceived stability of an afterimage during vestibular stimulation (Kaufman, 1974). Our own observations are that a foveal afterimage does appear to move in darkness during vestibular stimulation, but that its motion is not striking.

Aside from the contradictory reports on afterimage motion in darkness during involuntary eye movement, reported motion of an afterimage must be viewed circumspectly, since perceived motion can be generated without vestibular stimulation by attending to one side or the other 
of a stabilized image (Cushman, Tangney, Steinman, \& Ferguson, 1984; Kommerell \& Taeumer, 1972). In addition, reflex vestibular eye movements increase in amplitude when an afterimage is visible in an otherwise dark field (Yasui \& Young, 1976). Thus, additional evidence bearing on whether or not efference-copy signals accompany involuntary as well as voluntary eye movements is desirable.

Several authors have implicitly or explicitly questioned the neural level at which efference-copy signals are generated, by postulating that such information accompanies only voluntary (presumably cortical), and not involuntary or reflex (presumably subcortical), eye movement (Evanoff \& Lackner, 1987; Hoernsten, 1979; Post \& Leibowitz, 1985; Whiteside, Graybiel, \& Niven, 1965). By assuming that efference copy is available only for voluntary eye movements, an explanation is provided for the illusory displacement and motion of visual targets that can occur during involuntary eye movements. For example, the illusory motions of visual targets that occur during per-rotary or caloric nystagmus are accounted for by the retinal-image motions produced by the nystagmus, if they are not offset or canceled by concurrent internal signals of eye movement. Similarly, Hoernsten (1979) concluded that efference-copy signals exist neither for the pathological nystagmus in patients with acute vestibular neuritis nor for the accompanying offset of average eye position in darkness, and thereby explained these patients' inaccurate reports of the direction of isolated visual targets in an otherwise dark field.

Evidence that the brain is informed about involuntary eye movements comes from a study in which eye position could be correctly returned to straight ahead following vestibular eye movements induced by body rotation (Hansen \& Skavenski, 1977). However, Bridgeman, Lewis, Heit, and Nagle (1979) suggested that motor and perceptual systems receive different information about eye movements. Thus, perceptual reports suggesting that efference copy fails to offset retinal-image shifts during involuntary eye movement might arise because only the motor system, and not perception, is informed about such eye movements.

The purpose of this study was to evaluate whether or not eye-position information, presumably as efference copy, is available during optokinetic afternystagmus (OKAN), an involuntary eye movement that persists in darkness following optokinetic stimulation. To do so, the perceived direction of briefly presented visual targets was measured, in the first experiment by unseen manual pointing and in the second experiment by pointing made under visual control.

\section{METHOD}

\section{Apparatus and Stimuli}

Full-field optokinetic stimulation was produced by the rightward rotation of a cloth drum, within which the subject sat. The drum was $1.5 \mathrm{~m}$ in diameter and consisted of alternate $1-\mathrm{cm}$-wide white and gray (contrast $=40 \%$ ) vertical stripes. At the subject's view- ing distance of $70 \mathrm{~cm}$, each stripe subtended a visual angle of $0.82^{\circ}$. A small lightbulb, suspended from the drum's hub, illuminated the white stripes so that their luminance was about $1.2 \mathrm{~cd} / \mathrm{m}^{2}$.

Horizontal eye movements were recorded with a Model 200 EyeTrac (available from Gulf + Western), which compares reflected infrared light from the nasal and temporal limbi. The infrared sensors and light source were mounted on a blank spectacle frame, strapped behind the subject's head to minimize movement. Head position was maintained by a large, foam head-rest on the back of the subject's chair, into which the subject's head fit snugly. This chair was sufficiently tall so that the subject's feet did not reach the ground.

The eye-position signals were fed through an 8-bit A/D converter (Connecticut Microcomputer) to a Commodore 4032 PET computer. A machine-language computer program sampled eye position at intervals of less than $1 \mathrm{msec}$ and identified quick phases of nystagmus according to a velocity criterion. After an electronic signal from the experimenter, the program identified the next quick phase, waited for 150 to $500 \mathrm{msec}$ (depending upon the value input during initialization of the program), and then presented a red laser target for approximately $20 \mathrm{msec}$ by activating a noiseless shutter. The laser target was projected from a mirror galvanometer (General Scanning Model G330) onto the outside surface of the cloth drum, through which the target was visible to the subject. The position of the target on the drum was varied pseudorandomly within a range of about $15^{\circ}$ right and left of straight ahead, according to the voltage input to the mirror galvanometer. The straight-ahead direction was defined by the last position of the continuously visible laser target (placed in the subject's apparent median plane) during the eye-position calibration before each period of optokinetic stimulation. Calibration (from sequential fixation at three or more positions of the laser spot) was repeated after each trial as well. The horizontal positions of the left and right eyes, and the position and time of presentation of the laser target, were recorded on an oscillograph (Hewlett-Packard Model 7404A) for off-line analyses.

\section{Procedure}

A trial began with a 30-sec period of rightward optokinetic stimulation at a drum velocity of $45 \% / \mathrm{sec}$, followed by complete darkness. This duration and velocity of stimulation has been reported to produce good OKAN in normal subjects (Cohen, Henn, \& Raphan, 1981; LaFortune, Ireland, Jell, \& DuVal, 1986a, 1986b). Indeed, in preliminary trials, primary OKAN (in the same direction as the previous stripe motion) was elicited in 25 of 27 normal subjects, with an average initial velocity (measured $5 \mathrm{sec}$ after optokinetic stimulation ceased) of $9.8 \% \mathrm{sec}$ and an average duration of $40 \mathrm{sec}$. As noted previously, substantial individual variability existed in the velocity and duration of OKAN responses (Waespe, Huber, \& Henn, 1978) and, after repeated stimulation within a session, OKAN tended to reduce (Cohen, Matsuo, \& Raphan, 1977). A few seconds after the offset of the optokinetic stimulus, the experimenter signaled the computer to look for a nystagmus quick phase and to trigger presentation of the laser target. In Experiment 1 , as soon as the subject detected the laser target, he/she closed both eyes and pointed bimanually with a penlight to indicate the target's perceived direction. Using landmarks on the outside of the drum, the experimenter noted the direction of pointing (to $0.5 \mathrm{~cm}$ ), which was later compared with the target's physical direction. The subject was instructed to extinguish the penlight and then open his/her eyes for additional target presentations, which continued until the primary (rightward) phase of OKAN ceased (see Figure 1).

Trials in Experiment 2 differed in that the subject kept both eyes open while pointing in the perceived direction of the flashed target and, hence, directed the penlight under visual guidance. Because fixation of stationary visual contours greatly reduces or eliminates primary OKAN even after darkness is restored (Cohen et al., 1977; 


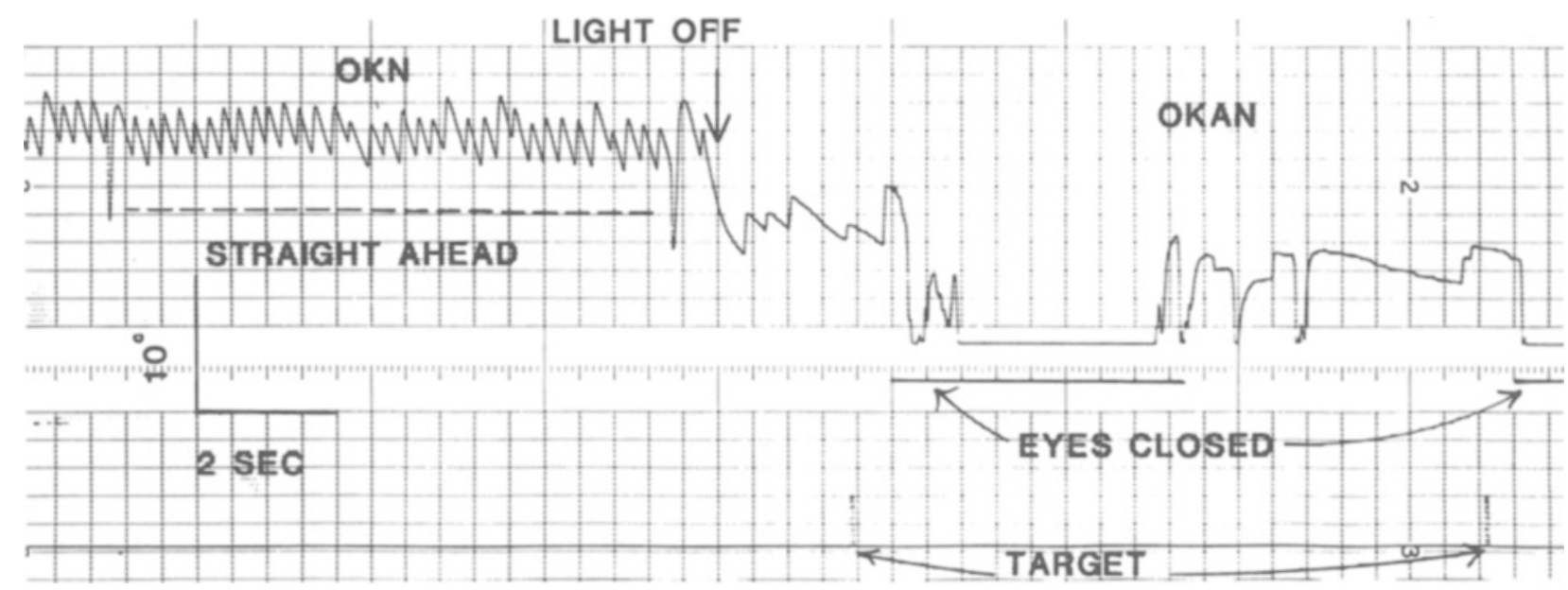

Figure 1. Horizontal eye position (upper tracing) for Subject J.E. in Experiment 1 during optokinetic nystagmus (OKN) and optokinetic afternystagmus (OKAN) subsequent to light off. Downward deflections represent rightward eye movement, except for the large downward deflections that signify blinks (cf. 1 sec prior to light off). Presentations of the target during OKAN occurred during upward deflections in the lower tracing. Horizontal lines between the upper and lower tracing identify periods of eye closure while the subject made unseen pointing responses. The straight-ahead direction of the eyes was identified from fixation of a stationary target during calibration prior to OKN and following OKAN. Calibration bars indicate 2-sec duration and a $10^{\circ}$ change of eye position.

Waespe et al., 1978), in Experiment 2 only a single target presentation was made after each 30 -sec period of optokinetic stimulation.

Control trials, in which pointing was made without prior optokinetic stimulation, were conducted for all but 1 subject (J.E.) either before OKAN trials or on a separate experimental day. On these trials, target presentation was triggered by spontaneous saccadic eye movements that the subject made in darkness.

The subjects were instructed to maintain their gaze straight ahead at all times (except when pointing in the direction of the target in Experiment 2), but to attempt to keep the moving stripes clear when they were visible. Although the experiments were performed binocularly, no subject reported diplopia.

Different groups of 7 subjects participated in each of Experiments 1 and 2. All had normal corrected visual acuity and ocular motility. During rightward optokinetic stimulation, all subjects reported the sensation of leftward self-motion (vection) which, except for Subject C.T. (see Results), faded rapidly in darkness. Recordings made during control trials in the dark indicated no spontaneous nystagmus, except for Subject S.T. in Experiment 1. In darkness, this subject exhibited a small-amplitude, right-beating jerk nystagmus with an average slow-phase velocity of less than $1 \% / \mathrm{sec}$ but, nevertheless, demonstrated rightward OKAN following periods of optokinetic stimulation. Subject N.T. was tested on 2 separate days for Experiment 2; otherwise, all experimental trials were completed in a single 1-1.5-h session.

\section{RESULTS}

If, during OKAN, accurate signals of eye position are available to be combined with information about a target's retinal-image location, then pointing responses to visual stimuli should be essentially veridical. On the other hand, if no eye-position signals accompany involuntary eye movements during OKAN, then pointing should reflect the target's retinal-image location, because internally represented eye position remains straight aheadwhere the subjects were attempting to hold their gaze. To be accurate, each pointing response would have to be com- pensated by adding the eye position measured at the instant of target presentation.

The results for Experiment 1 (Figure 2) illustrate that open-loop (unseen) pointing was indeed quite veridical for targets presented during OKAN. The best-fit line to the data has a slope of 1.08 and an intercept of 0.23 , not significantly different from the values for slope (1.11) and intercept $(0.04)$ found during control trials, when there was no OKAN [multiple regression for coincidence of two regression lines using a dummy-group variable, $F(2,163)$ $=0.15, p=.86 \mathrm{j}$. Moreover, the correlation of 0.92 between target position and pointing on OKAN trials is similar to the value obtained during control trials $(0.95)$ and is significantly higher $[t(100)=7.53, p<.001]$ than the correlation of 0.72 between target position and compensated pointing responses (pointing + eye position). Indeed, pointing errors during OKAN were uncorrelated with eye position $(r=0.03)$, indicating that the addition of measured eye position to compensate pointing responses actually introduced extraneous variability and reduced the correlation with target position (see the Appendix). Besides being uncorrelated with eye position, pointing was also uncorrelated with the velocity of eye movement $(r$ $=0.13$ ) which, on OKAN trials, averaged $4 \% \mathrm{sec}$ to the right.

Similar results were obtained for closed-loop pointing (made under visual control) in Experiment 2. During OKAN, pointing was again accurate on average (Figure 3), the best-fit regression line having both a slope and an intercept of 1.01. The correlation of target position with pointing was $r=0.93$, comparable to that during control trials $(r=0.95)$ and was significantly better than the correlation with compensated pointing $[r=0.79$, $t(60)=5.22, p<.001]$. Like Experiment 1 , the bestfit regression lines did not differ significantly from coin- 


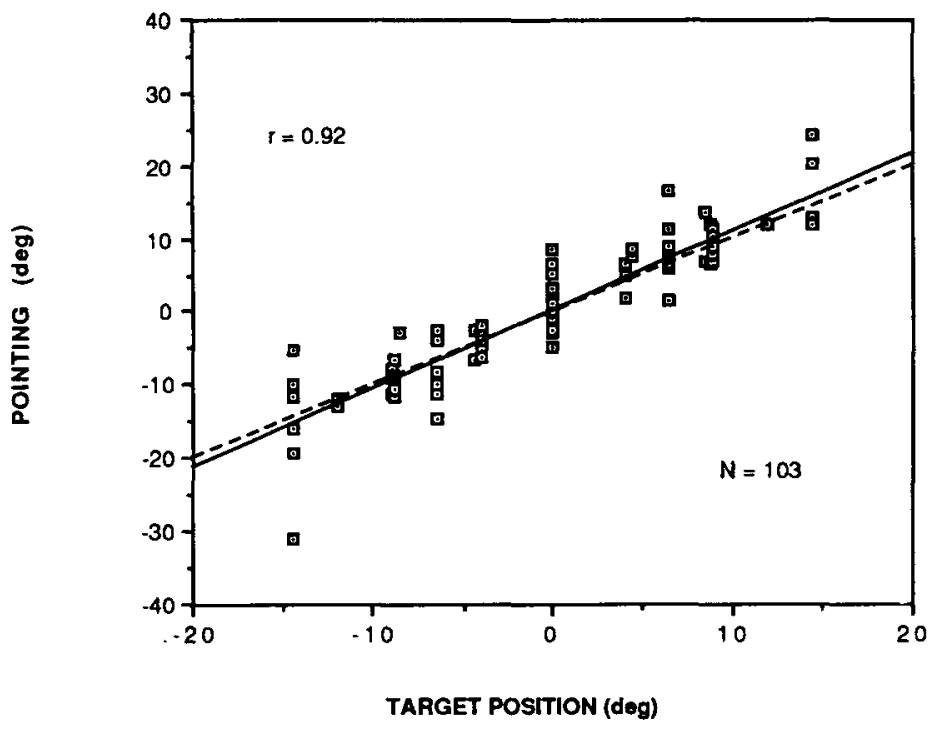

OPEN-LOOP COMPENSATED POINTING DURING OKAN

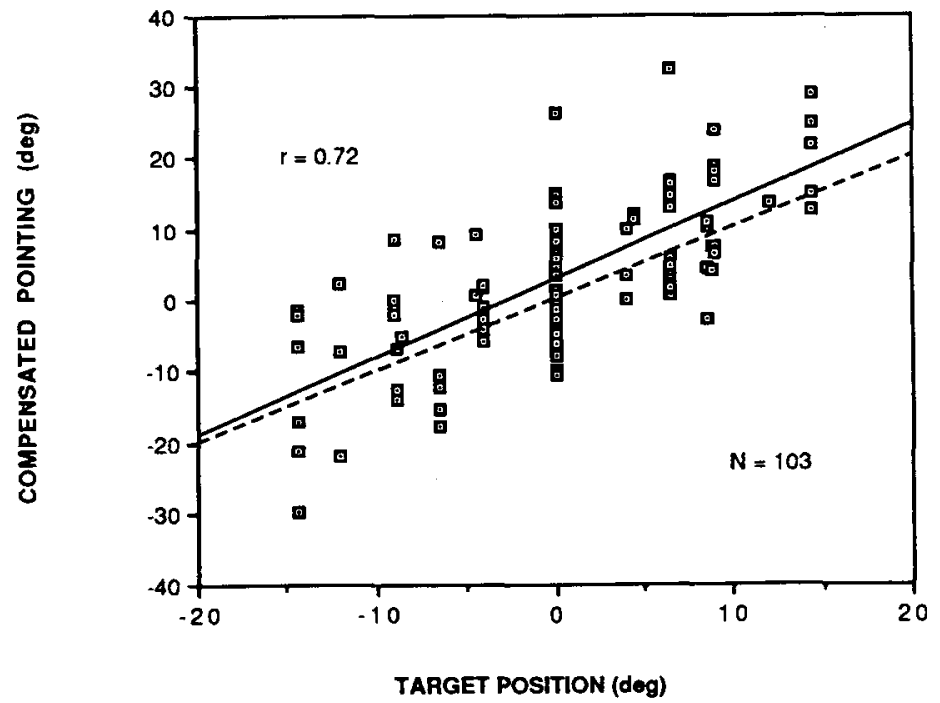

Figure 2. Upper panel-Unseen (open-loop) pointing responses for 7 subjects plotted against the position of the flashed target in Experiment 1 (positive = rightward). Lower panel-The relationship with target position when each pointing response is compensated by adding measured eye position, under the hypothesis that no extraretinal signal exists for involuntary OKAN. Solid lines are the best fit to the data points; dashed lines indicate veridical pointing.

cidence for OKAN and control trials $[F(2,110)=2.52$, $p=.09$ ). Again, pointing errors were uncorrelated with eye position $(r=0.03)$ and eye velocity $(r=-0.16)$, which averaged $6.1 \%$ sec to the right on OKAN trials.

Subject C.T. was unusual in that, subsequent to each of the first four periods of optokinetic stimulation in Experiment 2 , she perceived vivid leftward self-motion that persisted during OKAN in the dark. Pointing responses on these four trials were excluded from analysis because her pointing error averaged $13.3^{\circ}$ rightward of the physical direction of the targets. These large, systematic pointing errors could not be attributed to the absence of eyeposition information because, when pointing was compensated by adding measured eye position, the average 


\section{CLOSED-LOOP POINTING DURING OKAN}

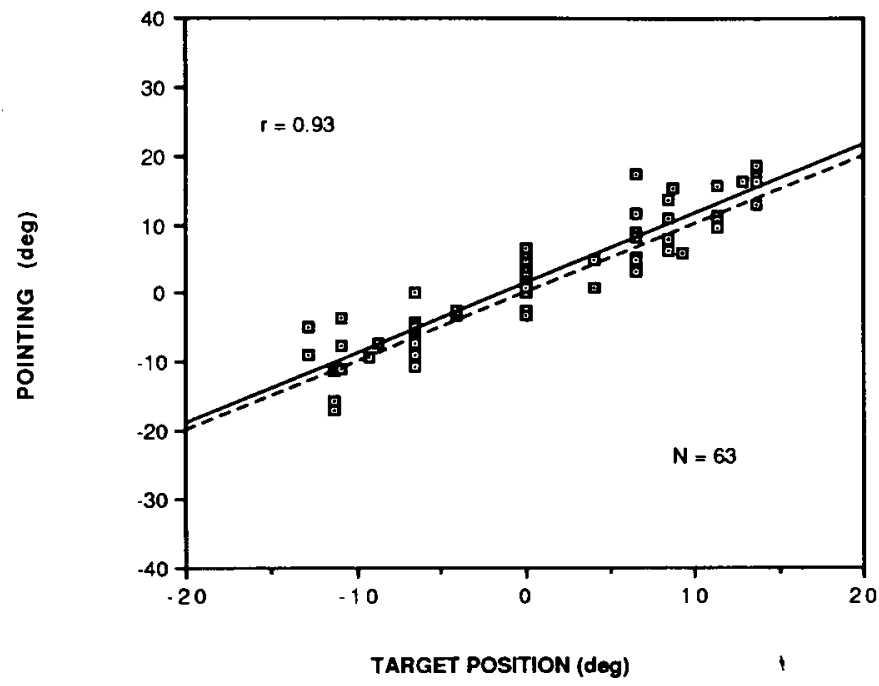

CLOSED-LOOP COMPENSATED POINTING DURING OKAN

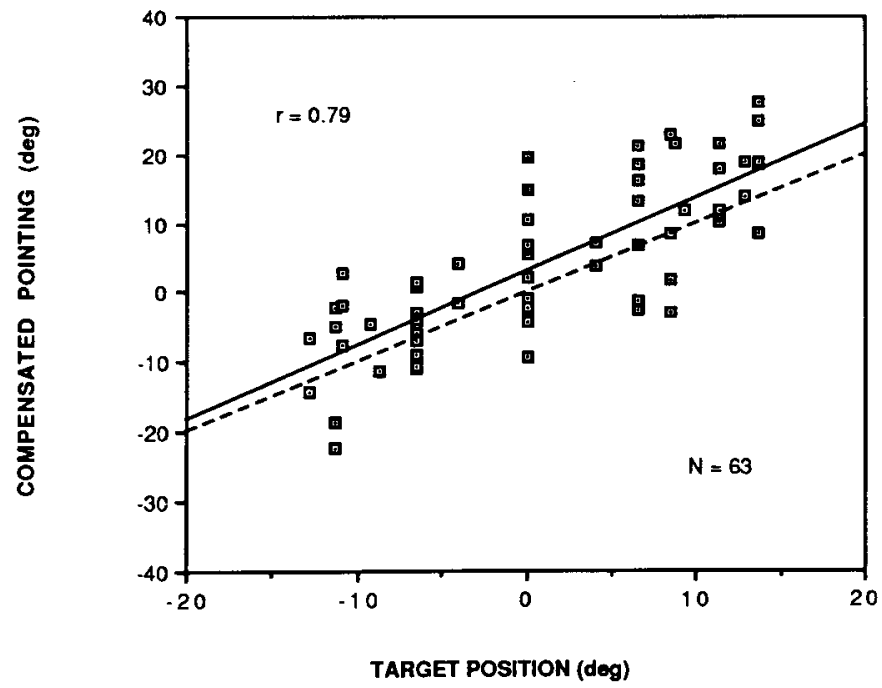

Figure 3. Upper panel-Pointing under visual control (closed-loop) plotted against the position of the flashed target for the 7 subjects in Experiment 2. Lower panel-The relationship with target position when pointing responses are compensated by adding measured eye position. Solid lines are the best fit to the data points; dashed lines indicate veridical pointing.

error remained essentially unchanged at $14.2^{\circ}$. Our explanation for Subject C.T.'s large rightward pointing errors during trials on which she experienced a strong sensation of vection is that her pointing incorporated a "correction" for her illusory leftward self-motion which, in the interval between the presentation of the target and her pointing response, was perceived as turning her away from the target's direction.

\section{DISCUSSION}

The results of these experiments demonstrate that extraretinal information about eye position is available during OKAN, presumably in the form of efference copy. Because OKAN is involuntary and is mediated subcortically, the results are consistent with the attribution by von Holst and Mittelstaedt (1950/1971) of efference copy to 
a low level in the motor pathway. Information about eye position during OKAN is accurate, at least within the range of eye positions (about $30^{\circ}$ ) and velocities (to about $16 \% \mathrm{sec}$ ) examined, as shown by the fact that the best-fit regression lines of pointing versus target position have slopes very close to 1.0 in both experiments. Previous investigators have concluded that extraretinal signals slightly underestimate actual eye position during voluntary fixation in different directions of gaze, but in those studies, a greater range of eye rotation was considered (Hill, 1972; Morgan, 1978).

Residual variability about the best-fit lines of pointing versus target position was comparable during OKAN and control trials (fixation straight ahead in the dark), indicating that the variability of the eye-position signal is comparable under these two conditions. Pointing errors on OKAN trials had an average within-subject variability (standard deviation) of $2.45^{\circ}$ in Experiment 1 and $2.69^{\circ}$ in Experiment 2, substantially less than the average within-subject variability of eye positions $\left(5.28^{\circ}\right.$ and $5.63^{\circ}$ in Experiments 1 and 2, respectively). A large portion of the residual pointing errors must be attributed to imprecision of manual pointing responses which, in prior studies involving pointing to remembered target locations, was on the order of $2^{\circ}$ (Brown, Knauft, \& Rosenbaum, 1948; Honda, 1984).

The very similar results obtained in Experiment 1 (in which pointing was unseen) and Experiment 2 (in which pointing was made under visual control) provide evidence against the notion that only motor systems have accurate information about eye position during involuntary eye movements. Even though manual pointing responses were employed in both experiments, these are known to be dominated by visual input when the two are placed in conflict (Hay, Pick, \& Ikeda, 1965; Rock \& Harris, 1967). Thus, the task in Experiment 2-to direct the penlight beam to the remembered location of a flashed target-is tantamount to asking the subject to match successively the perceived directions of two visual targets.

How can the present results be reconciled with those of prior investigators who reported that visual targets are localized erroneously during involuntary eye movements? Consider, in particular, the results of Hoernsten (1979), who reported that patients with an involuntary lateral deviation of gaze, resulting from acute vestibular neuritis, adjusted a luminous target approximately to the direction of gaze deviation in order for it to appear straight ahead. These errors of directionalization occurred when the target was presented in darkness, but not with room illumination. Hoernsten concluded from these results that his patients lacked information about their involuntarily deviated eye position in darkness, but other interpretations are possible. First, the distinction between "straight ahead" and "directly where one is looking" is subtle (von Noorden, 1970), and Hoernsten's instructions to the patients may not have clearly distinguished these alternatives. Thus, the patients may have adjusted the target to where the fovea was directed in the dark, not because they lacked information about eye position, but because this was how they interpreted the task. Indeed, Hansen (1979) offered essentially this explanation to account for an observation suggesting that voluntary pursuit eye movements are grossly underrepresented by their extraretinal signals. This observation was that the nonpursued of two orthogonally moving targets is perceived to move along an illusory path consistent with its path of motion on the retina; however, Hansen found that his subjects accurately set the path of the nontracked target when instructions adequately specified that motion of the target was to be judged with respect to the subject and not with respect to the tracked target.

A second interpretation of Hoernsten's (1979) results is based on the premise that, when a patient's eyes deviate in the dark (say, to the left), the patient simultaneously receives vestibular information of self-rotation (to the right). The presence of an eye deviation and associated nystagmus (beating toward the primary gaze position) attest to the presence of neuritis-induced vestibular activity that signals rotation of the head in the direction opposite the eye deviation. If Hoernsten's patients attempted to locate the straight-ahead direction with reference to the (unseen) room, then they would be expected to adjust the luminous target in the direction of eye deviation in the dark, because vestibular-driven eye movements normally serve to maintain an unchanged eye position with respect to the external world during head rotation (Robinson, 1977). Hence, a fixed external target should be approximately where vestibular innervation directs the eyes; perceptually, it is irrelevant that this vestibular information is anomalous and the (suggested) accompanying sense of rotation is illusory. As when external factors interfere with voluntary eye movements (Matin et al., 1982; Stark \& Bridgeman, 1983), we presume (in agreement with Hoernsten) that room illumination provides information about visual contours that contradicts and largely overcomes the anomalous vestibular signals of rotation.

In the Results section, we advanced essentially this latter explanation to account for Subject C.T.'s substantial pointing errors on OKAN trials, during which she perceived herself as rotating. Thus, we suggest that the illusory motion and displacement of visual targets that can occur when involuntary eye movements are stimulated in normal subjects may be explained, at least partly, on the basis of the accompanying internal signals of self-motion. Clearly, the pattern of retinal stimulation that occurs during involuntary eye movement should also be considered as an explanatory factor for these illusions (e.g., see Byford, 1963).

\section{REFERENCES}

Bridgeman, B., Lewis, S., Heit, G., \& Nagle, M. (1979). Relation between cognitive and motor-oriented systems of visual position perception. Journal of Experimental Psychology: Human Perception \& Performance, 5, 692-700.

Brown, J. S., KNauft, E. B., \& Rosenbaum, G. (1948). The accuracy of positioning reactions as a function of their direction and extent. American Journal of Psychology, 61, 167-182. 
ByFord, G. H. (1963). Eye movements and the optogyral illusion. Aerospace Medicine, 34, 119-123.

Cohen, B., Henn, V., RApHan, T. (1981). Velocity storage, nystagmus, and visual vestibular interactions in humans. Annals of the New York Academy of Sciences, 374, 421-433.

Cohen, B., Matsuo, V., \& RaPhan, T. (1977). Quantitative analysis of the velocity characteristics of optokinetic nystagmus and optokinetic afternystagmus. Journal of Physiology, 270, 321-344.

Cushman, W. B., Tangney, J. F., Steinman, R. M., Ferguson, J. L. (1984). Characteristics of smooth eye movements with stabilized targets. Vision Research, 24, 1003-1009.

Evanoff, J. N., \& LACKNER, J. R. (1987). Influence of maintained ocular deviation on the spatial displacement component of the oculogyral illusion. Perception \& Psychophysics, 42, 25-28.

GOETHLIN, G. F. (1946). Entoptic analysis of vestibular nystagmus. Acta Otolaryngologica, 34, 230-245.

Graybiel, A., HuPP, D. I (1946). The oculogyral illusion: A form of apparent motion which may be observed following stimulation of the semicircular canals. Journal of Aviation Medicine, 17, 3-27.

GRUESSER, O.-J. (1986). Interaction between efferent and afferent signals in visual perception: A history of ideas and experimental paradigms. Acta Psychologica, 63, 3-21.

Guthrie, B. L., Porter, J. D., \& Sparks, D. L. (1983). Corollary discharge provides accurate eye position information for the oculomotor system. Science, 221, 1193-1195.

HANSEN, R. M. (1979). Spatial localization during pursuit eye movements. Vision Research, 19, 1213-1221.

Hansen, R. M., \& SKavenski, A. A. (1977). Accuracy of eye position information for motor control. Vision Research, 17, 919-926.

HaY, J. C., Pick, H. L., \& IkeDA, K. (1965). Visual capture produced by prism spectacles. Psychonomic Science, 2, 215-216.

HaYs, W. L. (1963). Statistics. New York: Holt, Rinehart \& Winston.

Hill, A. L. (1972). Direction constancy. Perception \& Psychophysics, $11,175-178$

HoERnsten, G. (1979). Constant error of visual egocentric orientation in patients with acute vestibular disorder. Brain, 102, 685-700.

HoNDA, H. (1984). Functional between-hand differences and outflow eye position information. Quarterly Journal of Experimental Psychology, 36A, 75-88.

HowARD, I. (1982). Human visual orientation. New York: Wiley.

KaufMaN, L. (1974). Sight and mind. New York: Oxford University Press.

Kommerell, G., \& TAeUmer, R. (1972). Investigation of the eye tracking system through stabilized retinal images. Biblioteca Ophthalmolog. ica, 82, 288-297.

LaFortune, S., Ireland, D. J., Jell, R. M., \& DuVAL, L. (1986a). Human optokinetic afternystagmus: Charging characteristics and stimulus exposure time dependence in the two-component model. Acto Otolaryngologica, 101, 353-360.

LAFortune, S., IRELAND, D. J., Jell, R. M., \& DUVAL, L. (1986b) Human optokinetic afternystagmus: Stimulus velocity dependence of the two-component decay model and involvement of pursuit. Acta Otolaryngologica, 101, 183-192.

Matin, L., Picoult, E., Stevens, J. K., Edwards, M., Young, D., \& MacArThur, R. (1982). Oculoparalytic illusion: Visual-field dependent spatial mislocalizations by humans partially paralyzed with curare. Science, 216, 198-201.

Morgan, C. L. (1978). Constancy of egocentric visual direction. Perception \& Psychophysics, 23, 61-68.

MorRison, L. C. (1984). Visual localization with eye movements: A review. Ophthalmic \& Physiological Optics, 4, 339-353.

Post, R. B, \& Leibowitz, H. W. (1985). A revised analysis of the role of efference in motion perception. Perception, 14, 631-643.

RoBinson, D. (1977). Vestibular and optokinetic symbiosis: An example of explaining by modelling. In R. Baker \& A. Berthoz (Eds.), Control of gaze by brainstem neurons (pp. 49-55). Amsterdam: Elsevier.

RoCK, I., HARRIS, C. (1967). Vision and touch. Scientific American, 216, 96-104.

Skavenski, A. A., Haddad, G., \& Steinman, R. M. (1972). The ex- traretinal signal for the visual perception of direction. Perception \& Psychophysics, 11, 287-290.

STARK, L., \& Bridgeman, B. (1983). Role of corollary discharge in space constancy. Perception \& Psychophysics, 34, 371-380.

von Holst, E., \& Mitrelstedt, H. (1971). The principle of reafference: Interactions between the central nervous system and the peripheral organs. In P. C. Dodwell (Trans.), Perceptual processing: Stimulus equivalence and pattern recognition (pp. 41-71). New York: Appleton-Century-Crofts. (Original work published 1950)

VON NOORDEN, G. K. (1970). Etiology and pathogenesis of fixation anomalies in strabismus: 3 . Subjective localization. American Journal of Ophthalmology, 69, 228-236.

Wade, N. J. (1978). Sir Charles Bell on visual direction. Perceprion, 7, 359-362.

W aespe, W., Huber, T., Henn, V. (1978). Dynamic changes of optokinetic after-nystagmus (OKAN) caused by brief visual fixation periods in monkey and man. Archiv fuer Psychiatrie und Nervenkrankheiten, 226, 1-10.

Whiteside, T. C. D., Graybiel, A., Niven, J. I. (1965). Visual illusions of movement. Brain, 88, 193-210.

YAsUI, S., \& Young, L. R. (1976). Eye movements during afterimage tracking under sinusoidal and random vestibular stimulation. In R. A. Monty \& J. W. Senders, (Eds.), Eye movements and psychological processes (pp. 33-37). Hillsdale, NJ: Erlbaum.

\section{APPENDIX}

In this paper, we argue that it is unnecessary to compensate subjects' pointing responses to targets presented during OKAN by adding measured eye position. We therefore need to account for the reasonably good correlations $(0.72$ in Experiment 1 and 0.79 in Experiment 2) obtained between target position (Tar) and compensated pointing on OKAN trials. Our analysis is based on an interpretation of the squared correlation coefficient as the proportion of variance of one variable (here, target position) accounted for by the other (compensated pointing; cf. Hays, 1963, pp. 501-502):

$$
r^{2}=\frac{\text { variance accounted for by correlation }}{\text { total variance }} .
$$

If it is assumed that pointing (Pt) is accurate during OKAN trials, except for random error (err), then

$$
r^{2}(\mathrm{Pt} \cdot \mathrm{Tar})=\frac{\operatorname{var}(\mathrm{Pt})-\operatorname{var}(\mathrm{err})}{\operatorname{var}(\mathrm{Pt})} .
$$

For compensated pointing (Ptc), measured eye position (Eye) is added to target position, so that

$$
\operatorname{var}(\mathbf{P t c})=\operatorname{var}(\mathbf{P t})+\operatorname{var}(\text { Eye })+\operatorname{var}(\text { err }) .
$$

However, eye position is uncorrelated with pointing error and contributes to total variance, but not the variance accounted for by correlation.

$$
r^{2}(\mathrm{Ptc} \cdot \mathrm{Tar})=\frac{\operatorname{var}(\mathrm{Pt})-\operatorname{var}(\mathrm{err})}{\operatorname{var}(\mathrm{Pt})+\operatorname{var}(\mathrm{Eye})} .
$$

If both sides are multiplied by $[\operatorname{var}(\mathrm{Pt})+\operatorname{var}(\mathrm{Eye})] / \mathrm{var}(\mathrm{Pt})$ and rearranged,

$$
\begin{aligned}
& r^{2}(\mathrm{Ptc} \cdot \text { Tar) } \\
& =\frac{\operatorname{var}(\mathrm{Pt})}{\operatorname{var}(\mathrm{Pt})+\operatorname{var}(\mathrm{Eye})} \cdot \frac{\operatorname{var}(\mathrm{Pt})-\operatorname{var}(\mathrm{err})}{\operatorname{var}(\mathrm{Pt})} .
\end{aligned}
$$


But $r^{2}(\mathrm{Pt} \cdot \mathrm{Tar})=[\operatorname{var}(\mathrm{Pt})-\operatorname{var}(\mathrm{err})] / \operatorname{var}(\mathrm{Pt})$, so that

$$
r^{2}(\mathrm{Ptc} \cdot \mathrm{Tar})=\frac{\operatorname{var}(\mathrm{Pt})}{\operatorname{var}(\mathrm{Pt})+\operatorname{var}(\mathrm{Eye})} \cdot r^{2}(\mathrm{Pt} \cdot \mathrm{Tar}) .
$$

Entering the values for $\operatorname{var}(\mathrm{Pt})$ and $\operatorname{var}(\mathrm{Eye})$ from Experiment 1 and using $r(\mathrm{Pt} \cdot \mathrm{Tar})=0.92$,

$$
\text { Expected } r^{2}(\mathrm{Ptc} \cdot \mathrm{Tar})=0.841 \cdot \frac{78.32}{78.32+53.44} .
$$$$
\text { Expected } r(\mathrm{Ptc} \cdot \mathrm{Tar})=0.71 \text {. }
$$

Similarly, for Experiment 2, in which $r(\mathbf{P t} \cdot \mathrm{Tar})=0.93$,

$$
\text { Expected } r^{2}(\operatorname{Ptc} \cdot \text { Tar })=0.863 \cdot \frac{85.01}{85.01+39.56} .
$$

Expected $r$ (Ptc $\cdot$ Tar $)=0.77$. revision accepted for publication July 11, 1989.)

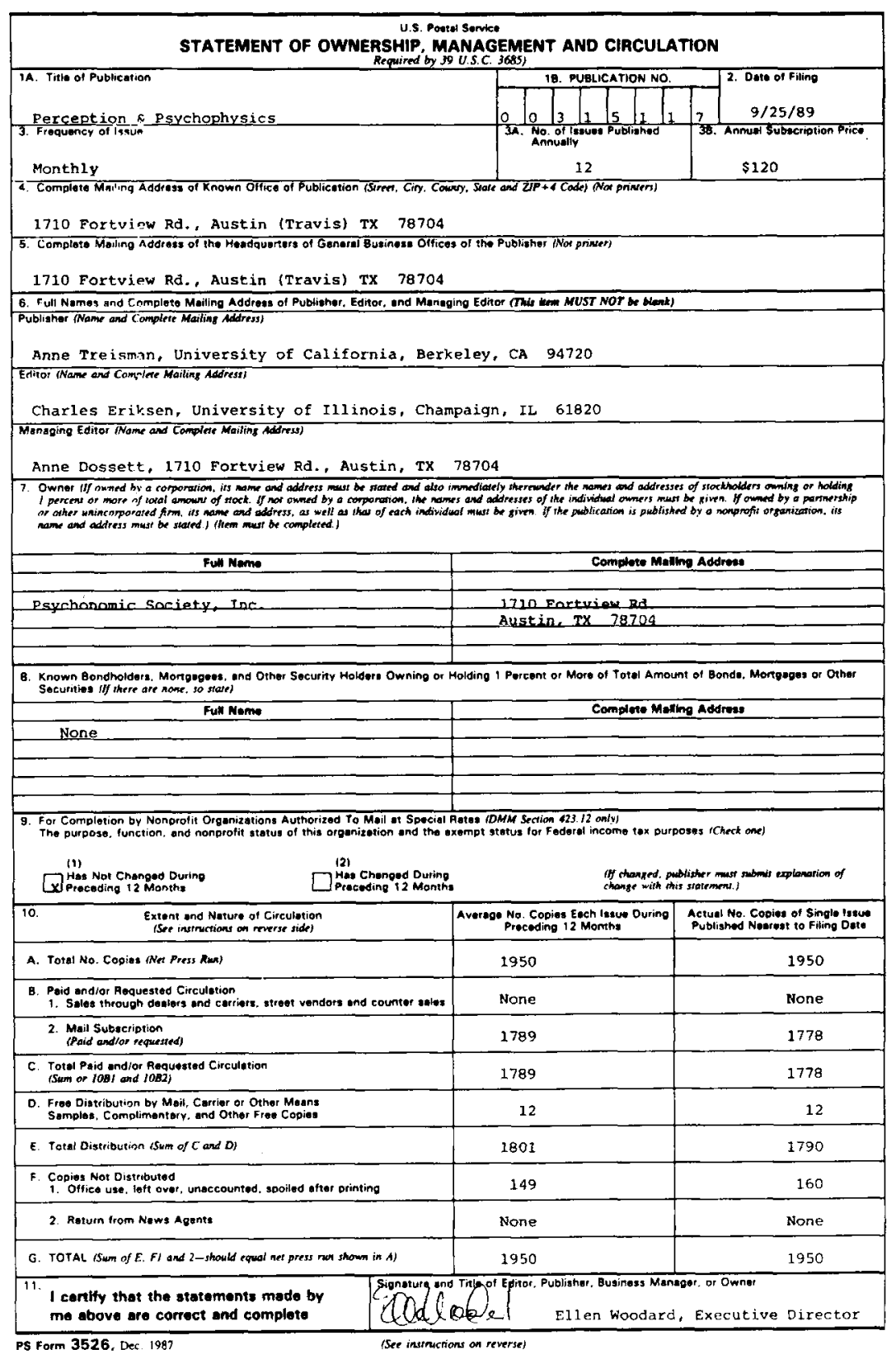

